\title{
EDITORIAL
}

\section{Special Issue on Biomedical Optics}

\author{
Kyung Min Byun
}

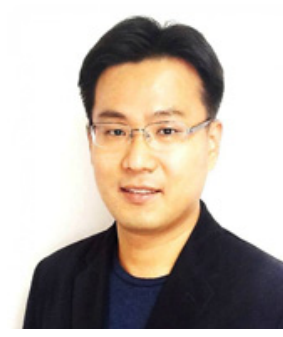

Woonggyu Jung

(C) The Korean Society of Medical \& Biological Engineering and Springer 2014

Biomedical optics is an interdisciplinary field of combining principles of optics with a variety of technologies in biology, biophysics, biochemistry, medicine, computer science and bio-related engineering. This field has been gaining a great interest due to unique characteristics of light, for example, high spatial resolution, three-dimensional and real-time detection, noninvasive or minimally invasive procedure and simple manipulation. Such light-based research encompasses a wide range of basic study, inspection, diagnostics and therapy of biological cells and tissues. In order to keep pace with rapid advancement and to introduce current research trends, we select biomedical optics as a theme of this Special Issue of Biomedical Engineering Letters. This Special Issue includes five review articles and one original research paper covering various state-of-the-art technologies.

The first paper entitled "The potential of naturally occurring lasing for biological and chemical sensors" by Choi and Kim introduces alternative biosensing methods of taking advantage of naturally occurring lasers, also known as

Kyung Min Byun $(\varangle)$

Kyung Hee University, Yongin, Republic of Korea

Tel : +82-31-201-3842 / Fax : +82-31-202-1723

E-mail : kmbyun@khu.ac.kr

Woonggyu Jung $(\square)$

Ulsan National Institute of Science and Technology, Ulsan, Republic of Korea

Tel : +82-52-217-2542

E-mail :wgjung@unist.ac.kr random lasers [1]. In random lasers, resonances are selfformed due to multiple scattering, leading to light amplification and coherent light generation in the presence of amplifying media. In this case, lasing emission can be unprecedentedly sensitive to subtle nanoscale perturbations. The authors address that random lasers can be an alternative yet superior physical mechanism for biosensors, because the random laser biosensing platform is simple and the detection strategy is straightforward.

The second paper entitled "Acoustic resolution photoacoustic microscopy" by Park and colleagues reviews the principle and system implementation of acoustic-resolution photoacoustic microscopy (AR-PAM) [2]. Note that, by combining information provided by light and acoustic properties, it is possible to realize high sensitivity and specificity, strong contrast of biological tissues, and relatively deep penetration beyond the depth limitation of traditional optical imaging. The authors also present several applications of AR-PAM to image biological tissues such as sentinel lymph nodes, lymphatic systems, bladders, gastrointestinal tracts, and whole body of small animals. Owing to its outstanding imaging capability, AR-PAM is anticipated to be applied for a variety of biomedical imaging researches.

The third paper entitled "Recent functional near infrared spectroscopy based brain computer interface systems: Developments, applications and challenges" is described by Phillips V and Kim [3]. Portable and convenient functional near infrared spectroscopy (fNIRS) can provide an enormous 
potential of implementing brain computer interface (BCI) systems. This review presents how fNIRS can be paired with another modality such as electroencephalography and function transcranial Doppler sonography to drastically improve a classification accuracy and how fNIRS-BCI systems are used to enhance human-robot interactions and assistive technologies. Remaining challenges in this field are also discussed as future research topics.

The fourth paper entitled "Plasmon based super resolution imaging for single molecular detection: Breaking the diffraction limit" by Choi and colleagues explains the principle of surface plasmon-mediated bioimaging method and introduces localized plasmon based super resolution imaging techniques to increase lateral and axial resolution below the diffraction limits [4]. Three novel imaging techniques based on field localization are introduced to increase a lateral resolution and additional three approaches based on extraordinary transmission and Förster resonance energy transfer are introduced to increase an axial resolution. Future direction towards surface plasmon based 3D spatiotemporal super resolution microscopy will allow to detect single molecule and track its dynamics.

The fifth paper entitled "Diagnostic fiber-based optical imaging catheters" written by Kim and colleagues pinpoints the recent development of fiber based optical imaging catheters with rotary scanning for diagnosing various diseases in luminal organs, including the coronary artery and esophagus [5]. High resolution fiber based imaging techniques, including optical coherence tomography and spectrally encoded confocal microscopy, combined with helical scanning and imaging catheters for the endoscopic procedures based on them are critically reviewed.

The last paper entitled "Design study on photoacoustic probe to detect prostate cancer using 3D Monte Carlo simulation and finite element method" is an original research article by El-Gohary and colleagues [6]. The authors propose an alternative photoacoustic probe based on transurethral laser illumination and validate its improvement using 3D prostate model and analytical methods. Placing light source inside the urethra and ultrasound transducer on the rectal wall is determined as an optimal combination to produce a high amplitude in pressure signal and a high signal-to-noise ratio in reconstructed image. It is expected that this study will be helpful in designing an optimized probe of photoacoustic imaging system for advancing the detection of prostate cancer.

Finally, we guest editors would like to express our gratitude to the contributing authors who review the promising technologies and report novel and original research in the field of biomedical optics. We would also like to acknowledge the support from the Managing Editor (Prof. Jae Sung Lee) and Editor-in-Chief (Prof. Sang-Hoon Lee) of the Biomedical Engineering Letters.

\section{REFERENCES}

[1] Choi SH, Kim YL. The potential of naturally occurring lasing for biological and chemical sensors. Biomed Eng Lett. 2014; 4(3):201-12.

[2] Park S, Lee C, Kim J, Kim C. Acoustic resolution photoacoustic microscopy. Biomed Eng Lett. 2014; 4(3):213-22.

[3] Phillips VZ, Kim JG. Recent functional near infrared spectroscopy based brain computer interface systems: Developments, applications and challenges. Biomed Eng Lett. 2014; 4(3):223-30.

[4] Choi J-R, Lee S, Kim K. Plasmon based super resolution imaging for single molecular detection: Breaking the diffraction limit. Biomed Eng Lett. 2014; 4(3):231-8.

[5] Kim JY, Lee MW, Yoo H. Diagnostic fiber-based optical imaging catheters. Biomed Eng Lett. 2014; 4(3):239-49.

[6] El-Gohary SH, Metwally MK, Eom S, Jeon SH, Byun KM, Kim T-S. Design study on photoacoustic probe to detect prostate cancer using 3D Monte Carlo simulation and finite element method. Biomed Eng Lett. 2014; 4(3):250-7. 